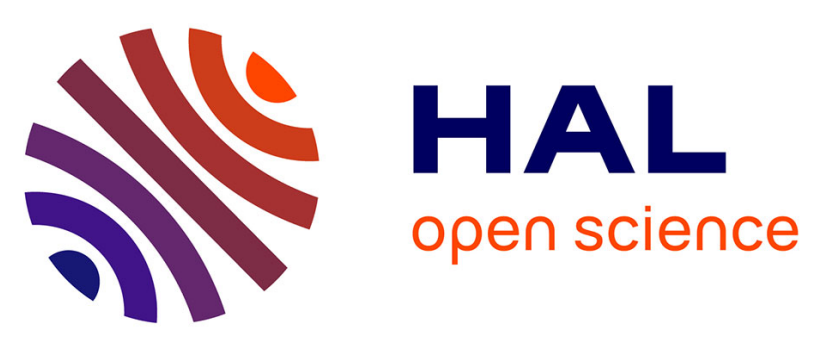

\title{
Improved evaluation of spin-polarization energy contributions using broken-symmetry calculations
}

Grégoire David, Nicolas Ferré, Georges Trinquier, Jean-Paul Malrieu

\section{To cite this version:}

Grégoire David, Nicolas Ferré, Georges Trinquier, Jean-Paul Malrieu. Improved evaluation of spinpolarization energy contributions using broken-symmetry calculations. Journal of Chemical Physics, 2020, 153 (5), pp.054120. 10.1063/5.0011734 . hal-02913985

\section{HAL Id: hal-02913985 \\ https://hal-amu.archives-ouvertes.fr/hal-02913985}

Submitted on 11 Aug 2020

HAL is a multi-disciplinary open access archive for the deposit and dissemination of scientific research documents, whether they are published or not. The documents may come from teaching and research institutions in France or abroad, or from public or private research centers.
L'archive ouverte pluridisciplinaire HAL, est destinée au dépôt et à la diffusion de documents scientifiques de niveau recherche, publiés ou non, émanant des établissements d'enseignement et de recherche français ou étrangers, des laboratoires publics ou privés. 


\title{
Improved evaluation of spin-polarization energy contributions using broken-symmetry calculations
}

Grégoire David, ${ }^{\dagger \S}$ Nicolas Ferré,${ }^{\dagger}$ Georges Trinquier, ${ }^{\ddagger}$ and Jean-Paul Malrieu ${ }^{t^{*}}$

\footnotetext{
${ }^{\dagger}$ Aix Marseille Univ, CNRS, ICR, 13397 Marseille, France

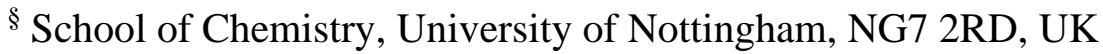

$\$$ Laboratoire de chimie et physique quantiques, IRSAMC-CNRS-UMR5626, Université Paul-Sabatier (Toulouse III), 31062 Toulouse Cedex 4, France
}

\begin{abstract}
Spin-polarization effects may play an important role in free radicals and in the magnetic coupling between radical centers. Starting from restricted open-shell calculations, i.e. a closed-shell description of the non-magnetic core electrons, a low-order perturbation expansion identifies the spin polarization contribution to the energy of mono-radicals and to singlet-triplet energy differences in diradicals. Broken-symmetry (BS) single-determinant calculations introduce only a fraction of spin polarization effects, and in a biased manner, since BS determinants are not spin eigenfunctions. We propose a simple technique to correctly evaluate spin-polarization energies by taking into account the effect of spin-flip components of one-hole one-particle excited configurations. Spin-decontamination corrections is shown to play a non-negligible role in BS evaluation of bond energies. The importance of spin-decontamination is illustrated on cases for which spin polarization is the leading contribution to the singlet-triplet gap, what characterizes twisted conjugated double-bonds and disjoint diradicals.
\end{abstract}

*Corresponding author (malrieu@ irsamc.ups-tlse.fr) 


\section{Introduction}

The spin-polarization phenomenon has been identified and interpreted since the end of the fifties by Mc Connell and other experimentalists. ${ }^{1-4}$ The key phenomenon concerned the contact term in ESR spectra of conjugated radical hydrocarbons. Their unpaired electron is described by a $\pi$ molecular orbital (MO) with zero density in the plane of the molecule, hence where protons lie. The experiments showed the existence of an interaction between electronic and nuclear spins. They further revealed that when the unpaired electron has $m_{S}=+1 / 2$ character, the spin density on in-plane protons is negative. This suggests that the two inner-shell non-magnetic (core) electrons which should occupy the same space MO according to the simplest representation (the $\sigma$-bond closed-shell core MOs) tend to occupy different space MOs according to their spin. This difference is due to the exchange field created by the unpaired electron, which acts differently on $\alpha$ and $\beta$ electrons of the bond electron pair. In other words, the core is subject to a spin polarization phenomenon.

The same phenomenon takes place in diradicals also, with two unpaired electrons essentially located in different regions of the molecule. The two electrons may be of parallel spins in a triplet state, or of opposite spins in a singlet state. The spin-polarization phenomenon may impact the energy difference between singlet and triplet states, ultimately modifying the magnetic coupling between the two magnetic centers. The most dramatic effect concerns the diradicals where the two magnetic orbitals are orthogonal, as it occurs for the $90^{\circ}$-twisted ethylene molecule, for which the singlet < triplet energy ordering is governed by the spin polarization contribution. ${ }^{5,6}$ But it is also the driving contribution in so-called "disjoint diradicals", where magnetic centers are connected through atoms where spin density presents a node, provided that the two SOMOs do not present a significant through-space hopping integral. ${ }^{7}$ This is not the case, however, in tetra-methylene-ethane (TME, also called diallyle) $\left(\mathrm{CH}_{2}\right)_{2} \mathrm{C}-\mathrm{C}\left(\mathrm{CH}_{2}\right)_{2}$ - a typical representative of this class. ${ }^{8-13}$ Here, allyl SOMOs are connected through their nodal sites, but they exhibit a through-space hopping integral, whatever dihedral angle between allyl groups, hence non-zero kinetic exchange. In the hereafter studied systems the SOMOs will differ by symmetry and the kinetic exchange contribution will be zero. Whatever type of diradicals, spin-polarization effects may contribute significantly to their magnetic coupling anyway. ${ }^{14}$

The simplest approximation for introducing this phenomenon in a quantum chemical calculation consists in starting from a restricted open-shell (RO) single determinant, where "core" electrons are constrained to be paired in closed-shell MOs. In a second step, this constraint is released and the "core" electrons can now occupy distinct orbitals for $\alpha$ and $\beta$ spins. This "unrestricted" single determinantal mean-field calculation, ${ }^{15,16}$ hereafter called "broken symmetry" (BS), proceeds by an energy minimization. ${ }^{17,18}$ Such calculations have two defects: (i) the corresponding wave functions are not eigenfunctions of the $S^{2}$ spin operator, they are "spin-contaminated", and (ii) these single-determinant calculations miss important corrections proceeding through double excitations from the RO determinant, which precisely restore the spin multiplicity. Both problems are internally related and have to be repaired. 
Of course, configuration interaction $(\mathrm{CI})$ calculations are possible, but expensive, and one may be tempted to reach reasonable estimates using low-cost single-determinant calculations. An approximate spindecontamination formula proposed by Yamaguchi is frequently used for diradicals, ${ }^{19-21}$ but it has no foundation to take care of spin-polarization effects, as shown in a previous paper. ${ }^{22}$ The present work introduces new usage of RO and BS solutions which enables one to avoid spin contamination and to incorporate the effect of double excitations.

Section 2 will recall the basic features of the spin polarization phenomenon for a mono radical. On the mono-radical problem, a perturbative expansion is presented and confronted to the content of energy change between the RO and BS mean-field solutions. This enables a spin-decontaminated evaluation of the spin-polarization energy contribution. More general and less approximated projection techniques have been proposed by Scuseria et al. ${ }^{23-25}$ but they are of more complex use. In single-bond breakings, the products of the reaction are free radicals, whose energy are lowered by spin polarization. A correct evaluation of this term may be important for a proper evaluation of the bond energy, as shown by our numerical study.

Section 3 briefly recalls the role of the spin-polarization correction in diradicals, on both singlet and triplet states, and on their energy difference. Exploiting the RO and BS energies of the $m_{S}=1$ and $m_{S}=0$ solutions, one proposes an evaluation of spin polarization contribution to the magnetic coupling, free from spin contamination problem and incorporating double spin-flip excitations corrections. This section presents numerical applications concerning the singlet-triplet gap on systems where kinetic exchange, i.e. the antiferromagnetic contribution brought by mixing with valence-bond (VB) ionic configurations, is either zero for symmetry reasons or negligible, as in disjoint diradicals. In these systems, spin decontamination significantly increases the singlet-to-triplet gap.

For both mono- and di-radicals, the derivation motivating the present method can be summarized as follows. (i) First, one considers the interaction between the RO determinant and the singly-excited determinants as a perturbation, and defines their contributions to the first-order wave-function and the second-order energy. (ii) Then, one shows how the same effects are treated as a spin-dependent relaxation of the core MOs in the BS single-determinant variational treatment. One establishes the connection between the perturbative and BS variational treatments. (iii) Next, one considers spin-flip excitations, generating determinants belonging to the same space-configurations as the singly-excited determinants. The perturbation expansion establishes the simple relations between the effects of single excitations and those of spin-flip excitations. The first-order wave function adding the single excitations and the spin-flip excitation is an eigenfunction of the $S^{2}$ operator. (iv) Last, one takes benefit of the relations between the BS calculation, the effect of the single excitations and the effect of the spin-flip excitations to propose a spin-decontaminated evaluation of the total spin polarization contribution to the energy from the spincontaminated BS calculation. 


\section{Spin polarization in monoradicals}

\section{A) Analytic derivation}

Let us start with the RO single determinant

$\Phi_{R}=\left|a \Pi_{k} k \bar{k}\right|$

where $a$ is the singly occupied MO, bearing the unpaired electron, and $k$ runs the closed-shell MOs. The mean-field Fock operator for the core electrons is

$F=h+\Sigma_{k}\left(2 J_{k}-K_{k}\right)+J_{a}-K_{a} / 2$

where $h$ is the mono-electronic operator, $J$ and $K$ coulomb and exchange operators respectively. If $r$ is a virtual MO, Brillouin's theorem imposes

$\left\langle\left(a_{r}^{+} a_{i}+a_{r}^{+} a_{i}\right) \Phi_{R}|H| \Phi_{R}\right\rangle=0$,

i. e. that all matrix elements between occupied and virtual orbitals are zero:

$\langle i|F| r\rangle=0$

Despite this condition, the $\Phi_{R}$ determinant interacts with singly-excited determinants, the excitation involving either the $\alpha$ spin electron of shell $i$

$\Phi_{i \rightarrow r}=\left|\operatorname{arri} \bar{\Pi}_{k \neq i} k \bar{k}\right|$

or the $\beta$ spin electron of the same shell

$\Phi_{\bar{i} \rightarrow \bar{r}}=\left|a \overline{i r} \Pi_{k \neq i} k \bar{k}\right|$.

The interaction between the RO single determinant and these singly excited determinants are opposite. For the $\alpha$ spin excitation, the exchange operator with the single electron is $-K_{a}$, not $-K_{a} / 2$ as appearing in the Fock operator above,

$\left\langle\Phi_{R}|H| \Phi_{i \rightarrow r}\right\rangle=\left\langle i\left|-K_{a} / 2\right| r\right\rangle$,

while for the $\beta$ spin excitation there is no exchange with the unpaired electron and $\left\langle\Phi_{R}|H| \Phi_{\bar{i} \rightarrow \bar{r}}\right\rangle=\left\langle i\left|K_{a} / 2\right| r\right\rangle$.

These interactions are governed by the exchange operator of singly-occupied orbital $a$, acting oppositely on $\alpha$ and $\beta$ spin singly-excited determinants. The interaction between the restricted determinant and these singly-excited determinants, acting as perturbers, may be treated as a perturbation. The perturbing 
operator is the interaction between single determinants, and it gives a second-order spin-polarization energy correction, due to single excitations only:

$$
E_{S P m}^{(2)}=2 \Sigma_{i, r} \frac{\left\langle i\left|K_{a} / 2\right| r\right\rangle^{2}}{F_{i i}-F_{r r}}=\frac{1}{2} \Sigma_{i, r} \frac{\left\langle i\left|K_{a}\right| r\right\rangle^{2}}{F_{i i}-F_{r r}} .
$$

Here the excitation energy from $i$ to $r$ has been approximated by the difference between the diagonal energies of the Fock operator, as usual in the so-called Moller-Plesset expansion. The BS single determinant is obtained by a spin-dependent relaxation of doubly occupied MOs, leading to the single determinant

$\Phi_{B S}=\left|a \Pi_{i} i^{\prime} i^{\prime \prime}\right|$

It is well-known that orbital relaxation and CI interaction between reference and singly-excited determinants are related. To the first order of perturbation, the $\alpha$ spin-orbital $i$ ' may be approximated as

$i^{\prime}=i-\Sigma_{r} \frac{\left\langle i\left|K_{a} / 2\right| r\right\rangle}{F_{i i}-F_{r r}} r$

and the $\beta$ spin-orbital $i$ " as

$i^{\prime \prime}=i+\Sigma_{r} \frac{\left\langle i\left|K_{a} / 2\right| r\right\rangle}{F_{i i}-F_{r r}} r$

The orbital relaxation of the BS solution may be approximated by a first-order expansion of the wave function:

$\left|\Phi_{B S}\right\rangle \approx\left|\Phi_{R}\right\rangle+\Sigma_{i, r} \frac{\left\langle i\left|-K_{a} / 2\right| r\right\rangle}{F_{i i}-F_{r r}}\left(\Phi_{i \rightarrow r}-\Phi_{i \rightarrow r}\right)$

Notice that the function $\Phi_{i \rightarrow r}-\Phi_{\bar{i}_{\rightarrow}-\bar{r}}$ is the product of an $m_{s}=0$ triplet excitation in the core by a doublet and is not an eigenfunction of the $S^{2}$ operator, which is a consistent defect of the BS wave function. The energy stabilization obtained by relaxing the core MOs, i.e. by going from RO to BS determinants, can be approximated to a $2^{\text {nd }}$ order energy correction as

$$
\left\langle\Phi_{B S}|H| \Phi_{B S}\right\rangle-\left\langle\Phi_{R}|H| \Phi_{R}\right\rangle=E_{B S}-E_{R} \approx E_{S P m}^{(2)}=2 \Sigma_{i, r} \frac{\left\langle i\left|K_{a} / 2\right| r\right\rangle^{2}}{F_{i i}-F_{r r}}
$$


One should not omit that other determinants of the same space part contribute to the energy, and restore the correct spin multiplicity, namely the so-called spin-flip determinants

$$
\Phi_{a \bar{i} \rightarrow r \bar{a}}=\left|\bar{a} \cdot r i \Pi_{k \neq i} k \bar{k}\right| \text {, }
$$

which changes the $m_{s}$ value from $1 / 2$ to $-1 / 2$, and creates an excitation to an $m_{s}=1$ triplet in the core.

These doubly-excited determinants interact with $\Phi_{R}$ by

$$
\left\langle\Phi_{R}|H| \Phi_{a i \rightarrow r a}^{-}\right\rangle=\left\langle i a\left|r_{12}^{-1} a\right| r\right\rangle=\left\langle i\left|K_{a}\right| r\right\rangle .
$$

In a Moller-Plesset definition of the zero-order Hamiltonian the denominator relative to the spin-flip determinant is the same as the one relative to the $i \rightarrow r$ single excitation, namely $F_{i i}-F_{r r}$. The contribution of the spin-flip excitations to the energy is

$$
E_{S P d}^{(2)}=\Sigma_{i, r} \frac{\left\langle i\left|K_{a}\right| r\right\rangle^{2}}{F_{i i}-F_{r r}} \approx 2\left(\left\langle\Phi_{B S}|H| \Phi_{B S}\right\rangle-\left\langle\Phi_{R}|H| \Phi_{R}\right\rangle\right)
$$

i.e. twice the energy lowering obtained when going from the RO to the BS solutions. This effect of double excitations pertains to the same physics as spin-polarization single excitations since they involve the same bielectronic integrals and the same spatial configuration. Working with spin-eigenstate configurations instead of single determinants would take the spin-flip determinants into account, as the total spinpolarization actually does. One may consequently write the total spin-polarization as

$$
E_{S P}^{(2)}=E_{S P d}^{(2)}+E_{S P m}^{(2)}=3\left(\left\langle\Phi_{B S}|H| \Phi_{B S}\right\rangle-\left\langle\Phi_{R}|H| \Phi_{R}\right\rangle\right)
$$

and the energy of the doublet ground state may finally be written as

$$
E=E_{R}+3\left(E_{B S}-E_{R}\right)=3 E_{B S}-2 E_{R} .
$$

Adding the spin flip determinants to the first-order wave function restores the $S^{2}$ eigenfunction,

$$
\left|\Psi^{1}\right\rangle \approx\left|\Phi_{R}\right\rangle+\Sigma_{i, r} \frac{\left\langle i\left|-K_{a} / 2\right| r\right\rangle}{F_{i i}-F_{r r}}\left(\Phi_{i \rightarrow r}-\Phi_{\bar{i} \rightarrow \bar{r}}-2 \Phi_{a \bar{i} \rightarrow r \bar{a}}\right),
$$

as it is a linear combination of doublet states.

\section{B) Impact on bond-breaking energies}

Breaking homolytically a covalent single bond leads to two free radicals:

$$
\mathrm{A}-\mathrm{B} \rightarrow \mathrm{A} \cdot+\cdot \mathrm{B}
$$


The corresponding binding energy is evaluated as $\mathrm{E}(\mathrm{A} \cdot)+\mathrm{E}(\mathrm{B} \cdot)-\mathrm{E}(\mathrm{A}-\mathrm{B})$. The spin polarization of the two radicals may contribute to the binding energy. If the $\mathrm{A}-\mathrm{B}$ molecule is not subject to symmetry breaking, its energy may be estimated from a closed-shell calculation within the mean field approximation. Consistently, energies of the free radicals may be calculated in one of the following formalisms: RO, BS, or spin-decontaminated description. It is interesting to evaluate the importance of the spin polarization contribution on a few basic examples of bond dissociation energies.

We considered two examples of $\mathrm{C}-\mathrm{C}$ single bonds breakings. The first one is the dissociation of ethane into two $\mathrm{CH}_{3}$ radicals, which adopt a planar geometry. Using the B3LYP exchange-correlation functional in density functional theory (DFT), ${ }^{26,27}$ the RO energy of the radical is -39.85232 a.u., while the unrestricted solution, at -39.85376 a.u., is $0.9 \mathrm{kcal} / \mathrm{mol}$ below. The spin decontamination correction would be three times larger, contributing to the $\mathrm{CC}$ bond energy by as much as $-5.4 \mathrm{kcal} / \mathrm{mol}$, which represents about five percent of a CC single-bond energy - a non-negligible quantity. ${ }^{28}$

In another example, we consider the dissociation of the $\mathrm{CC}$ bond connecting the two cyclopentadienyl rings in hypothetical bicyclopentadienyl (Scheme 1). The RO-DFT energy of each radical fragment is -193.51054 a.u. and the unrestricted solution is -193.51298 a.u., lying therefore 1.5 $\mathrm{kcal} / \mathrm{mol}$ below. The spin-decontaminated spin polarization energy here lowers the bond energy by as

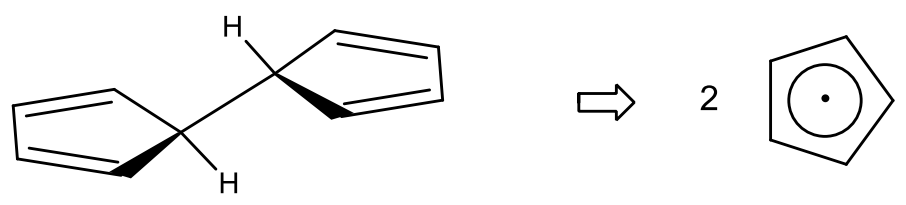

Scheme 1

much as $9.1 \mathrm{kcal} / \mathrm{mol}$, a rather large quantity in regard to the weak homolytic dissociation energy calculated in this system at $45.3 \mathrm{kcal} / \mathrm{mol}$ (not including ZPE corrections). Such a noticeable twentypercent lowering of the bond energy is not unexpected here, as the spin polarization effect is larger in conjugated $\pi$ radicals than in localized ones.

\section{C) Additional issues}

Spin densities. The spin contamination has important impact on the energy, but it does not have a similar importance for spin densities. Actually the spin density operator $\rho_{s}$ is mono-electronic. If we define $\Delta \Phi_{B S}=\Phi_{B S}-\Phi_{R}$,

the leading correction to spin density comes from the cross term 
$\Delta \rho_{s}^{(1)}=2\left\langle\Delta \Phi_{B S}\left|\rho_{s}\right| \Phi_{R}\right\rangle$,

which is a first-order correction. The spin-decontaminating corrections to the wave function are doubly excited with respect to $\Phi_{R}$ and do not contribute to the cross-term. It can only contribute to the spin density by second-order corrections, negligible in comparison to $\Delta \rho_{s}^{(1)}$. If one considers the spin density on an Atomic Orbital p, the coefficients of which in the MOs $i$ and $r$ are respectively $c_{i p}$ and $c_{r p}$, the firstorder correction, coming the singly excited determinants, is

$\Delta \rho_{p}^{(1)}=\sum_{i, r} 2 c_{i r} c_{r p}\langle r|K a / 2| i\rangle /\left(F_{i i}-F_{r r}\right)$.

The spin-flip determinants contribute to this spin density to the second order by

$\Delta \rho_{p}^{(2)}=\sum_{i, r}\left(c_{i p}^{2}+c_{r p}^{2}\right)\left(\langle r|K a| i\rangle /\left(F_{i i}-F_{r r}\right)\right)^{2}$.

In conjugated hydrocarbons, where the unpaired electron is in a $\pi \mathrm{MO}$, the spin-polarization of the $\sigma$ system proceeds through excitations from $\sigma$ bonding to $\sigma^{*}$ antibonding MOs which have the same amplitude on the $p \mathrm{AO}$, and the first-order contribution prevails largely. The only case where the contribution of the spin-flip component would prevail would concern excitation where either $c_{i p}$ or $c_{r p}$ would be negligible.

Dependence to $\left\langle\mathrm{S}^{2}>\right.$ deviation. It may be interesting to establish a connection between the spinpolarization energy and the deviation of $\left\langle S^{2}\right\rangle$, as calculated in the broken-symmetry single determinant, from its value for a pure doublet state, namely 0.75 . It is governed by the same summations over single excitations from core to virtual MOs. Assuming all $i \rightarrow r$ excitation energies are identical and equal to a value $\Delta$, according to a convenient closure approximation, the spin-polarization energy would be

$E_{S P}^{(2)}=\frac{3}{2} \Sigma_{i, r} \frac{\left\langle i\left|K_{a}\right| r\right\rangle^{2}}{\Delta}$

The BS determinant may be developed to first-order as

$\left|\Phi_{B S}\right\rangle=\left|\Phi_{R}\right\rangle+\Sigma_{i, r} \frac{\left\langle i\left|K_{a} / 2\right| r\right\rangle}{F_{i i}-F_{r r}}\left(a_{r}^{+} a_{i}-a_{r}^{+} a_{i}\right)\left|\Phi_{R}\right\rangle$.

The singly-excited components are a mixture of doublet and quartet states. The quartet state of the threeopen shells space function is

$\left|\Psi_{i r a}^{Q}\right\rangle=|\operatorname{core} .(\bar{i} r a+i \bar{r} a+i \bar{r} \bar{a}) / \sqrt{3}\rangle$

And its overlap with the BS determinant is

$$
\left\langle\Psi_{i r a}^{Q} \mid \Phi_{B S}\right\rangle=\frac{2}{\sqrt{3}} \frac{\left\langle i\left|K_{a} / 2\right| r\right\rangle}{F_{i i}-F_{r r}}=\frac{1}{\sqrt{3}} \frac{\left\langle i\left|K_{a}\right| r\right\rangle}{F_{i i}-F_{r r}} .
$$


The weight of the BS determinant on the quartet manifold is

$\sum_{i, r}\left(\left\langle\Psi_{i r a}^{Q} \mid \Phi_{B S}\right\rangle\right)^{2} \cong \frac{1}{3}\left(\frac{\left\langle i\left|K_{a}\right| r\right\rangle}{F_{i i}-F_{r r}}\right)^{2}$

the other components are doublets. The $S^{2}$ operator only acts inside a space configuration, and its value for a quartet is $15 / 4$. Performing the same closure approximation on energy denominators as the one we did for spin polarization energy, for the BS determinant the deviation from 0.75 of $\left\langle S^{2}\right\rangle$ is

$<S^{2}>_{B S}-3 / 4=\left\langle\Phi_{B S}\left|S^{2}\right| \Phi_{B S}\right\rangle-3 / 4=\Sigma_{i, r} \frac{\left\langle i\left|K_{a}\right| r\right\rangle^{2}}{3 \Delta^{2}} \cdot \frac{15}{4}=\frac{5}{4} \Sigma_{i, r} \frac{\left\langle i\left|K_{a}\right| r\right\rangle^{2}}{\Delta^{2}}$.

Equations (4) and (5) make clear the relation between the spin-polarization energy and the deviation of the mean value of the $S^{2}$ operator from its expected eigenvalue. The numerators are identical, but the excitation energies relative to the processes responsible for spin polarization also are specific of the molecule. From (4) and (5) one gets an evaluation of the mean energy of the excitations involved in the spin-polarization process:

$\Delta=\frac{5}{6} \frac{E_{S P}^{(2)}}{\left(<S^{2}>_{B S}-3 / 4\right)}$.

This relation has been applied to a set of typical monoradicals (Table 1). As expected, the so-calculated mean excitation energies are lower in conjugated hydrocarbons than in saturated ones, and they decrease with the extent of electronic delocalization, as particularly manifest in the linear polyenyl series.

Geometry alteration. One may wonder to which extent spin-decontamination could impact the calculated geometries of monoradicals. As done for singlet states of diradicals, ${ }^{30}$ an extrapolation procedure could be envisaged to get spin-decontaminated geometries by exploiting the optimized geometries obtained at RO level and from BS determinants. This possibility is however tempered by the fact that geometry changes between the two RO and BS solutions are quite negligible.

\section{Spin polarization in diradicals}

\section{A) Analytic derivation.}

In their simplest description, the two single electrons are described by two localized and orthogonal singly-occupied molecular orbitals (SOMO), $a$ and $b$. The calculation of a RO configuration, approximating either the singlet or the triplet state, provides such SOMOs. The experience shows that at this level the SOMOs of the two solutions are almost identical. ${ }^{31,32}$ 
In a RO description, the $m_{S}=0$ reference function is written as a combination of two determinants

$\Psi_{a b}^{1}=\left|\Pi_{k} k \bar{k} \cdot(b \bar{a}+a \bar{b})\right| / \sqrt{2}$.

Alternatively, in the BS approach, one may consider that one starts from the $m s=0$ determinant

$\Phi_{a \bar{b}}=\left|\Pi_{k} k \bar{k} \cdot a \bar{b}\right|$

with the same frozen core. Its energy can be obtained from those of the restricted open-shell calculations on $\Psi_{a b}^{1}$ and of the corresponding triplet state $\Psi_{a b}^{3}=\left|\Pi_{k} k \bar{k} \cdot(b \bar{a}-a \bar{b})\right| / \sqrt{2}$. The value of the direct exchange $2 K_{a b}$ between the two unpaired electrons in their singly-occupied MOs, a ferromagnetic contribution may be obtained from the energy difference between the two spin eigenfunctions of the same space configuration

$2 K_{a b}=\left\langle\Psi_{a b}^{1}|H| \Psi_{a b}^{1}\right\rangle-\left\langle\Psi_{a b}^{3}|H| \Psi_{a b}^{3}\right\rangle$,

quantities given by standard codes. The energy of the single determinant $\Phi_{a \bar{b}}=\left|\Pi_{k} k \bar{k} \cdot a \bar{b}\right|$ is equal to $E_{a \bar{b}}=\left\langle\Phi_{a \bar{b}}|H| \Phi_{a \bar{b}}\right\rangle=\left(\left\langle\Psi_{a b}^{1}|H| \Psi_{a b}^{1}\right\rangle+\left\langle\Psi_{a b}^{3}|H| \Psi_{a b}^{3}\right\rangle\right) / 2$

One may now consider the spin relaxation of the core orbitals, while keeping unmodified the magnetic orbitals, which leads to a BS determinant

$\Phi_{U, a \bar{b}}=\left|\Pi_{k} k^{\prime} \overline{k^{\prime \prime}} \cdot a \bar{b}\right| \cdot$

As done for the mono radical, we shall identify the relaxation energy treated by the BS calculation to the second-order perturbation energy resulting from the interaction between the reference determinant and the determinants obtained by single excitations on the top of the reference. To first order, the BS description incorporates the effect of the determinants

$\Phi_{k \rightarrow \bar{r}, a \bar{b}}=\left|\Pi_{i \neq k} i \bar{i} k \bar{r} \cdot a \bar{b}\right|$ and

$\Phi_{k \rightarrow r, a \bar{b}}=\left|\Pi_{i \neq k} \bar{i} \cdot \bar{k} \cdot \bar{k} \cdot a \bar{b}\right|$,

which stabilize the energy $E_{U, a \bar{b}}$ of $\Phi_{U, a \bar{b}}$ by the quantity

$E_{S P m}^{(2)}=\sum_{k, r} 2 \frac{\left\langle k\left|\left(K_{a}-K_{b}\right) / 2\right| r\right\rangle\left\langle r\left|\left(K_{a}-K_{b}\right) / 2\right| k\right\rangle}{F_{k k}-F_{r r}}=\sum_{k, r} \frac{1}{2} \frac{\left\langle k\left|\left(K_{a}-K_{b}\right)\right| r\right\rangle^{2}}{F_{k k}-F_{r r}} \cong E_{U, a \bar{b}}-E_{a \bar{b}}$

Full consideration of the effect of $k \rightarrow r$ excitations on the top of the singlet configuration, must include the spin-flip determinants which change the $m_{s}$ value of the electrons in singly-occupied MOs and in the singly-excited core, 
$\Phi_{k \rightarrow \bar{r},}^{S F}=\left|\Pi_{i \neq k} \bar{i} \bar{i} \cdot \bar{k} r . a b\right|$

$\Phi_{\bar{k} \rightarrow r,}^{S F}=\left|\Pi_{i \neq k} \bar{i} k k r \cdot \bar{a} \bar{b}\right|$

Both of them interact with the reference by the integral $\left\langle r\left|K_{b}-K_{a}\right| r\right\rangle / \sqrt{ } 2$, so that the $2^{\text {nd }}$-order correction brought by the spin-flip determinants is equal to

$$
E_{S P S f}^{(2)}=\sum_{k, r} \frac{\left\langle k\left|\left(K_{a}-K_{b}\right)\right| r\right\rangle^{2}}{F_{k k}-F_{r r}}=2 E_{S P m}^{(2)} .
$$

One sees that the full spin-polarization contribution to the energy stabilization of the singlet state is given by

$$
{ }^{1} E_{S P}^{(2)}=\sum_{k, r} \frac{3 / 2\left\langle k\left|K_{a}-K_{b}\right| r\right\rangle^{2}}{F_{k k}-F_{r r}} .
$$

Accordingly, the total spin-polarization energy for the singlet is three times larger than its evaluation from the single determinant evaluation

$$
{ }^{1} E_{S P}^{(2)}=3\left(E_{U, a \bar{b}}-E_{a \bar{b}}\right) .
$$

In the absence of kinetic exchange contribution, the energy of the spin-decontaminated singlet is given by $E_{s}=\left\langle\Psi_{a b}^{1}|H| \Psi_{a b}^{1}\right\rangle+{ }^{1} E_{S P}^{(2)}$.

The restricted description of the $m_{S}=1$ triplet state is

$\Phi_{R}^{3}=\left|\Pi_{k} k \bar{k} \cdot a b\right|$

Let us call its energy

$E_{R}^{3}=\left\langle\Phi_{R}^{3}|H| \Phi_{R}^{3}\right\rangle$.

The Fock operator for the closed-shell MOs is $F=h+\sum_{K}\left(2 J_{k}-K_{k}\right)+J_{a}-K_{a} / 2+J_{b}-K_{b} / 2$.

The unrestricted single determinant

$$
\Phi_{U}^{3}=\left|\Pi_{k} k^{\prime} \overline{k^{\prime \prime}} . a b\right|
$$

introduces the spin polarization of the closed shell under the effect of the singly-excited determinants of the type

$$
\Phi_{\bar{k} \rightarrow \bar{r}}^{3}=\left|\Pi_{i \neq k} i \bar{i} k \bar{r} \cdot a b\right| \text { and } \Phi_{k \rightarrow r}^{3}=\left|\Pi_{i \neq k} i \bar{i} . r \bar{k} \cdot a b\right|
$$

and to $2^{\text {nd }}$-order, the energy lowering may be approximated as 
$E_{U}^{3}-E_{R}^{3}=\sum_{k, r} 2 \frac{\left\langle k\left|\left(K_{a}+K_{b}\right) / 2\right| r\right\rangle\left\langle r\left|\left(K_{a}+K_{b}\right) / 2\right| k\right\rangle}{F_{k k}-F_{r r}}=\sum_{k, r} \frac{1}{2} \frac{\left\langle k\left|\left(K_{a}+K_{b}\right)\right| r\right\rangle^{2}}{F_{k k}-F_{r r}}$

This introduces the effect of the single excitations only, and it must add the effect of doubly-excited determinants involving a spin-flip between core and magnetic MOs. ${ }^{30}$ The determinant

$\Phi_{\bar{k} \rightarrow r, b \rightarrow \bar{b}}^{3}=\left|\Pi_{i \neq k} i \bar{i} k r . a \bar{b}\right|$

interacts with the reference through the integral $\left\langle r\left|K_{b}\right| k\right\rangle$, while the determinant

$\Phi_{\bar{k} \rightarrow r, a \rightarrow \bar{a}}^{3}=\left|\Pi_{i \neq k} i \bar{i} k r \cdot b \bar{a}\right|$.

interacts with the reference through the integral $\left\langle r\left|K_{a}\right| k\right\rangle$. They lead to a $2^{\text {nd }}-$ order correction due to spinflip determinants

${ }^{3} E_{S P s f}^{(2)}=\sum_{k, r} \frac{\left\langle k\left|K_{a}\right| r\right\rangle^{2}+\left\langle r\left|K_{b}\right| k\right\rangle^{2}}{F_{k k}-F_{r r}}$.

One may rewrite the full spin-polarization energy lowering for the triplet as

${ }^{3} E_{S P}^{(2)}=\sum_{k, r} \frac{1 / 2\left\langle k\left|K_{a}-K_{b}\right| r\right\rangle^{2}+\left\langle r\left|K_{a}+K_{b}\right| k\right\rangle^{2}}{F_{k k}-F_{r r}}$.

It is interesting that one may express the full spin polarization of the triplet state from the energy lowering of the single determinants:

$$
{ }^{3} E_{S P}^{(2)}=E_{U, a \bar{b}}-E_{a \bar{b}}+2\left(E_{U}^{3}-E_{R}^{3}\right) .
$$

The final energy of the triplet state may be written

$$
E_{T}=\left\langle\Psi_{a b}^{3}|H| \Psi_{a b}^{3}\right\rangle+{ }^{3} E_{S P}^{(2)}
$$

Equations (8) and (10) take into account the contributions of one-hole one-particle (1h-1p) excited determinants which are omitted in the unrestricted single-determinant calculations and which must be formally introduced to restore spin symmetry. These simple expressions should give a good approximation to the energy coming out of the CI calculation, running over all determinants of the $1 \mathrm{~h}-1 \mathrm{p}$ configurations, and may be considered as spin-contamination free. Notice that the unrestricted solutions are those obtained by freezing magnetic orbitals, according to the strategy of decomposition of magnetic couplings developed by Coulaud et $a l .^{31,32}$ The unrestricted $m_{s}=0$ solutions usually introduces a delocalization of magnetic orbitals $a$ and $b$, and consequently of ionic components responsible for the socalled kinetic exchange, a frequently dominant antiferromagnetic contribution not considered in the present work. 


\section{B) Applications to disjoint diradicals, torsional barriers, and isomerization energies}

The singlet-to-triplet gap of most anti-ferromagnetic diradicals (i.e. those having a singlet ground state) is governed by the interplay of three main physical contributions. ${ }^{33}$ Starting from the definition of two orthogonal magnetic orbitals $a$ and $b$, as given by a closed-shell core description (equation 6), one may distinguish: (i) a ferromagnetic direct exchange, (ii) an antiferromagnetic "kinetic exchange", due to the ionic VB component in the singlet state, and (iii) the spin polarization contributions of both states, whose sign is ruled by the topology of chemical connection between magnetic centers. For sake of simplicity we shall only consider systems where, for symmetry reasons, the kinetic exchange is zero. In this case, symmetry breaking is only due to spin polarization. In general, mixing with ionic VB component in an unrestricted single determinant is responsible for another symmetry breaking, and it is difficult to disentangle the two effects.

Disjoint diradicals are systems in which the two unpaired electrons occupy two magnetic orbitals $a$ and $b$ which have a small or zero interaction. ${ }^{7}$ The direct exchange $K_{a b}$ is then very small and the extradiagonal matrix element of the Fock operator between the two localized magnetic orbitals is close to zero, $\left(\left|t_{a b}\right| \approx 0\right)$. As the energy difference $U$ between ionic and neutral VB components is large, the kinetic exchange $-4\left(t_{a b}\right)^{2} / U$, is very small too. Commonly, disjoint diradicals exhibit two magnetic MOs connected by their nodal positions, i.e. through atoms where the SOMOs have zero coefficients. Provided that $t_{a b}$ develops over hopping integrals between sufficiently distant atoms (this is not the case in TME), the leading contribution to magnetic coupling is then the spin polarization mechanism. It is ruled by intraatomic preference to high spin multiplicity, and by spin alternation between adjacent atoms - spin-ordering effects plainly expressed by Ovchinnokov's rule. ${ }^{34}$

Other special diradicals are those where $a$ and $b$ belong to different irreducible representations, i.e. when $t_{a b}=0$ for symmetry reasons. A simple and famous example for this class is ethylene in its $90^{\circ}$ twisted geometry (Scheme 2, left), where the two unpaired electrons occupy $2 p_{\mathrm{x}}$ and $2 \mathrm{p}_{\mathrm{y}}$ orthogonal orbitals. Kollmar and Staemmler have shown why the spin-polarization correction is larger in the singlet state than in the triplet state, and why this antiferromagnetic correction prevails over the ferromagnetic direct exchange. ${ }^{5}$ The differential spin-polarization mechanism here goes through $\sigma \rightarrow \sigma^{*}$ single excitations relative to the central $\mathrm{CC}$ bond, and only acts on the singlet state for symmetry reasons. Let us detail the results for this system and its variant twisted styrene (Scheme 2, right). 

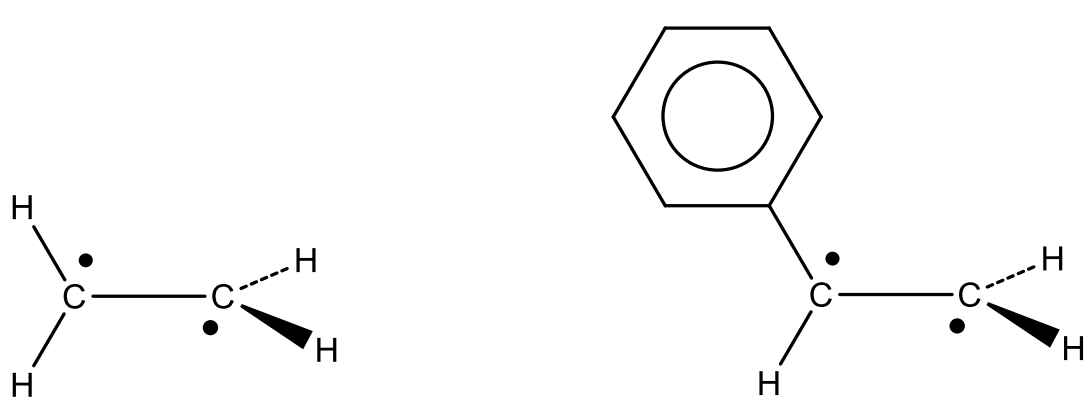

Scheme 2

Taken from RO-DFT calculations, the direct exchange $K_{a b}$ in twisted ethylene is calculated at $K_{a b}=0.00066$ a.u.. Comparing directly the BS energies for $m_{s}=1$ and $m_{s}=0$, which leads to an energy difference of 0.00138 a.u. $(0.9 \mathrm{kcal} / \mathrm{mol})$ in favor of the singlet, is not fair since one omits the lack of a $K_{a b}$ term in the singlet energy, and makes use of spin-contaminated spin-polarization energies. After spin decontamination, the spin polarization energy of the triplet $(-0.00801$ a.u.) is weaker than that of the singlet (-0.01207 a.u.), and the resulting energy difference between singlet and triplet is finally 1.7 $\mathrm{kcal} / \mathrm{mol}$. In this case, the correction to the ST gap is close to the classical multiplication by two of the difference between $m_{s}=1$ and $m_{s}=0$ UDFT solutions. ${ }^{22}$ At this geometry, the spin-decontaminated estimate of the vertical ST gap $(1.7 \mathrm{kcal} / \mathrm{mol})$ is in excellent agreement with that given by an accurate multireference CI calculation $(1.5 \mathrm{kcal} / \mathrm{mol}) .{ }^{35} \mathrm{We}$ can put to profit this improved energy to estimate a spindecontaminated rotational barrier. As can be seen in Table 2, bottom, the spin-decontamination correction lowers the rotational barrier by $4.7 \mathrm{kcal} / \mathrm{mol}$, corresponding to a seven-percent reduction of the raw barrier. $^{36}$

In ground-state twisted styrene, from a value of $K_{a b}$ calculated at 0.00044 , one gets a correction for the ST gap of $0.6 \mathrm{kcal} / \mathrm{mol}$, lowering it to $1.2 \mathrm{kcal} / \mathrm{mol}$ (cf Table 2). As in the preceding case, and not unexpectedly, it corresponds, here also, to a multiplication by a factor of two. The correction brought to the torsional barrier around $\mathrm{C}=\mathrm{C}$ by the spin decontamination is a decrease of $6.1 \mathrm{kcal} / \mathrm{mol}$, corresponding to an eleven-percent lowering, giving a final barrier at $48 \mathrm{kcal} / \mathrm{mol}$.

Further strictly disjoint diradicals can be conceived, such as those obtained by spirannically coupling polyenic radicals. As the spiro attachment takes place through a common saturated carbon atom where the SOMOs of two radicals have zero amplitude, this generates a disjoint diradical. If in addition the two radical moieties are in orthogonal planes, the diradical will be strictly disjoint, the hopping integral between the two SOMOs being zero for symmetry reasons. ${ }^{37}$ We have examined the case of two coupled pentadienyl radicals lying on a spiro-undecane frame (Scheme 3). The-direct exchange integral is here 


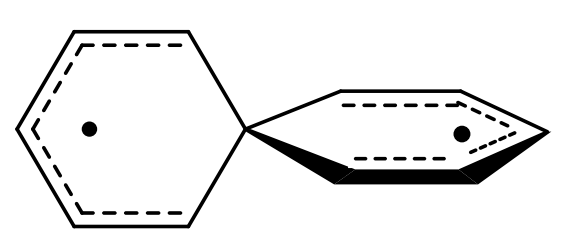

Scheme 3

0.00090 a.u. The spin polarization energy of the singlet (-0.02023 a.u.) is now lower than that of the triplet (-0.02094 a.u.), and the final energy difference is in favor of the triplet by $1.0 \mathrm{kcal} / \mathrm{mol}$. This is larger than what would suggest the simple energy difference between the BS solutions $(0.8 \mathrm{kcal} / \mathrm{mol})$, but the factor of two is here no longer appropriate. These examples show the importance of spin decontamination of spin polarization energy, at least when it becomes of the same order of magnitude as the direct exchange and in the absence of kinetic exchange.

As a last example, let us consider the energy difference between singlet closed-shell para-xylylene and its diradical isomer meta-xylylene in its triplet ground state (Scheme 4), and ask to which extent spinpolarization impacts this energy difference, before and after spin decontamination. In the restricted formalism, the para isomer is more stable than the meta isomer by $25.8 \mathrm{kcal} / \mathrm{mol}$. Spin polarization of course diminishes this difference, which only concerns the meta compound. Unrestricted treatment of the meta compound reduces the energy difference to $21.1 \mathrm{kcal} / \mathrm{mol}$. According to equation (6), spin decontamination significantly increases the spin-polarization correction from $4.7 \mathrm{kcal} / \mathrm{mol}$ to 11.7 $\mathrm{kcal} / \mathrm{mol}$, so that the energy difference between the two isomers finally falls to $14.1 \mathrm{kcal} / \mathrm{mol}$. Far from an exaustive study on xylylene isomerism, this exemple calls attention to the role of spin polarization and its correct treatment when comparing energies of closed-shell and open shell isomers.<smiles>C=c1ccc(=C)cc1</smiles>

para

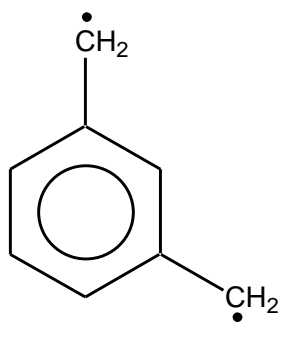

meta

Scheme 4 


\section{Conclusion}

In electronic structure calculations where spin-polarization may play an important role, as with mono- and polyradicals, as well as with transition states of most chemical reactions, one cannot rely on the RO formalism, which misses this effect. Its most accurate treatment consists in performing a perturbative or a variational expansion of the multi-determinant wave function. In this route, it is indeed possible to identify the excitations responsible for the spin-polarization effect. The corresponding electronic configurations belong to the class of the $1 \mathrm{~h}-1 \mathrm{p}$ excited configurations. Although simpler than the variational treatment, the perturbative expansion of their effect remains quite expensive since it requires the calculation of numerous bi-electronic integrals. BS approaches remaining in the single-determinant formalism are of much easier usage, and their simplicity explains their popularity, especially for the study of large molecular systems. But the resulting wave functions are not eigenfunctions of the $S^{2}$ operator, they only incorporate the effect of single excitations on the top of the reference restricted determinants and miss the effect of the doubly excited counterparts, which involve a spin flip in both the magnetic orbitals and in the core. Introducing these spin-flipped determinants restores $S^{2}$ eigenfunctions. The present work exploits the internal link between the coefficients of the singly excited determinants and those of the spinflipped determinants to exploit fully the unrestricted calculations.

The present work has presented first a convenient recipe which furnishes, through equation (3), a simple evaluation of the spin-decontaminated spin-polarization energy in free radicals. Numerical illustrations show the impact of the so-evaluated energies on the calculated bond energies when bond breaking leads to two free radical moieties. Then the spin-polarization energy of diradicals was considered, for both the triplet and the singlet eigenstates. Again the effect of the spin-flipped determinants was taken into account. Simple expressions, exploiting $m_{s}=1$ and $m_{s}=0$ UDFT single determinants, have been derived to produce spin-decontaminated spin-polarization energies, through equations (8) and (10). The numerical studies have been focused on systems where the so-called kinetic exchange is zero for symmetry reasons, and where spin-polarization is the leading factor governing singlet-to-triplet gaps. They show the importance of spin-decontamination on torsional barriers around double bonds in conjugated hydrocarbons. The numerical computations were performed in the DFT frame, but the developments apply to unrestricted Hartree-Fock calculations as well.

A further work will propose a general procedure for the treatment of those diradicals where both spin polarization and kinetic exchange contribute to the singlet-to-triplet gap. In this novel procedure, evaluations of these contributions will be free from spin contamination. Moreover, as it will also take into account the fact that spin polarization only concerns the neutral VB components of the singlet wave function, hence some non-additivity of kinetic exchange and spin polarization, it should thus correct the defects of Yamaguchi's formula ${ }^{19-21}$ and of our previous proposals..$^{22,31,32}$ 
Acknowledgment. This work was funded by French CNRS.

Availability of data. The data that support the findings of this study are available from the corresponding author upon reasonable request. 


\section{References and notes}

(1) B. Venkataraman and G. K. Fraenkel, J. Chem. Phys. 1956, 24, 737.

(2) H. M. Mc Connell, J. Chem. Phys. 1956, 24, 764.

(3) R. Bernsohn, J. Chem. Phys. 1956, 24, 1066.

(4) S. I. Weissman, J. Chem. Phys. 1956, 25, 890.

(5) H. Kollmar and W. Staemmler, Theor. Chim. Acta 1978, 48, 223.

(6) A.F. Voter, M. M. Goodgame, and W. A. Goddard III, Chem. Phys. 1985, 98, 7-14.

(7) M. Abe, Chem. Rev. 2011, 113, 7011-7088.

(8) J. A. Berson, Acc. Chem. Res. 1997, 30, 238-244.

(9) M. Filatov and S. Shaik, J. Phys. Chem. A 1999, 103, 8885-8889.

(10) E. Rodríguez, M. Reguero, and R. Caballol, J. Phys. Chem. A 2000, 104, 6253-6258.

(11) Z. D. Pozun, X. Su, and K. D. Jordan, J. Am. Chem. Soc. 2013, 135, 13862-13869.

(12) M. Barborini and E. Coccia, J. Chem. Theory Comput. 2015, 11, 5696-5704.

(13) L. Veis, A. Antalík, Ö. Legeza, A. Alavi, and J. Pittner, J. Chem. Theory Comput. 2018, 14, 2439-2445.

(14) J.-P. Malrieu, R. Caballol, C. J. Calzado, C. de Graaf and N. Guihéry, Chem. Rev. 2014, 114, 429.

(15) J. A. Pople and R. K. Nesbet, J. Chem. Phys. 1954, 22, 571-572.

(16) G. Berthier, J. Chim. Phys. 1954, 51, 363-371.

(17) A. P. Ginsberg, J. Am. Chem. Soc. 1980, 102, 111.

(18) L. Noodleman, J. Chem. Phys. 1981, 74 , 5737.

(19) K. Yamaguchi , H. Fukui and T. Fueno, Chem. Lett. 1986, 625-628.

(20) K. Yamaguchi , Y. Takahara, T. Fueno and K. N. Houk, Theor. Chim. Acta 1988, 73, 337-364.

(21) S. Yamanaka, M. Okumura , M. Nakano and K. Yamaguchi, J. Mol. Struct. THEOCHEM 1994, 310, 205-218.

(22) N. Ferré, N. Guihéry, and J.-P. Malrieu, Phys. Chem. Chem. Phys. 2015, 17, 14375-14382.

(23) T. Takahashi and G. Scuseria, J. Chem. Phys. 2011, 134, 064101. 
(24) C. A. Jiménez-Hoyos, T. M. Henderson, T. Tsuchimochi, and G. E. Scuseria, J. Chem. Phys. 2012, 136, 164109.

(25) P. Rivero, C. A. Jiménez-Hoyos, and G. E. Scuseria, J. Phys. Chem A 2013, 117, 8973-8080.

(26) All calculations are performed at the UB3LYP/6-311G** level, using the Gaussian 09 package. ${ }^{27}$ Full geometry optimizations are carried out up to energy gradients lower than $10^{-5}$ a.u. Cartesian coordinates for all optimized geometries are available upon request.

(27) M. J. Frisch, G. W. Trucks, H. B. Schlegel, G. E. Scuseria, M. A. Robb, J. R. Cheeseman, G. Scalmani, V. Barone, B. Mennucci, G. A. Petersson, et al., Gaussian 09, Revision D.01, Gaussian, Inc., Wallingford, CT, 2013.

(28) At the present level of description, and neglecting ZPE corrections, the CC dissociation energy in ethane is calculated at $93.3 \mathrm{kcal} / \mathrm{mol}$, The spin-decontamination correction would therefore lower this value to $87.9 \mathrm{kcal} / \mathrm{mol}$. The agreement with experimental bond dissociation enthalpy in ethane at $0 \mathrm{~K}$ $(87.9 \mathrm{kcal} / \mathrm{mol}),{ }^{29}$ is of course fortuitous, given the treatment modesty.

(29) Ruscic, J. Phys. Chem A 2015, 119, 7810-7837.

(30) G. Trinquier and J.-P. Malrieu, J. Phys. Chem. A 2012, 116, 10864-10869.

(31) E. Coulaud, N. Guihéry, J.-P. Malrieu, D. Hagebaum-Reignier, D. Siri and N. Ferré, J. Chem. Phys. 2012, 137, 114106.

(32) E. Coulaud, J. P. Malrieu, N. Guihéry and N. Ferré, J. Chem. Theory Comput. 2013, 9, 3429.

(33) C. J. Calzado, J. Cabrero, R. Caballol, and J.-P. Malrieu, J. Chem. Phys. 2002, 116, 2728-2747. The demonstration of the formula is given on page 2735 . There is a misprint in equation 46 , where a minus sign incorrectly appears in the last term of the equation.

(34) A. A. Ovchinnikov, Theor. Chim. Acta 1978, 47, 297-304.

(35) R. J. Buenker, S. Peyerimhoff, Chem. Phys. 1975, 9, 75-81.

(36) For recent theoretical works on ethylene torsional barrier, see: E. San-Fabian and F. Moscardo, $J$. Comput. Chem. 2014, 35, 1356-1363; S. S. Ray, U. S. Mahapatra, R. K. Chaudhuri, and S. Chattopadhyay, Comput. Theor. Chem. 2017, 1120, 56-78.

(37) G. Trinquier, N. Suaud, N. Guihéry, and J.-P. Malrieu, Chem. Phys. Chem. 2011, 12, 3020-3036. 
Table1: Confrontation of spin-polarization energies $\left(E_{S P}^{(2)}\right.$, in $\left.\mathrm{kcal} / \mathrm{mol}\right)$ and mean excitation energies of spin-polarizing single excitations $(\Delta$, in $\mathrm{eV})$ with calculated $\left\langle S^{2}\right\rangle$ in typical monoradicals.

\begin{tabular}{llrrr}
\hline & & & & \\
radical & & $\left\langle S^{2}\right\rangle$ & $E_{S P}^{(2)}$ & $\Delta$ \\
\hline methyl & & & & \\
tri-methyl-methyl & $\mathrm{CH}_{3}$ & 0.7535 & 2.7 & 27.83 \\
tri-phenyl-methyl (free $\left.D_{3}\right)$ & $\mathrm{C}_{3}\left(\mathrm{CH}_{3}\right)_{3}$ & 0.7540 & 2.8 & 25.71 \\
tri-phenyl-methyl (bridged $\left.C_{3 v}\right)$ & $\mathrm{C}_{3}\left(\mathrm{C}_{6} \mathrm{H}_{5}\right)_{3}$ & 0.7757 & 4.4 & 6.18 \\
cyclopentadienyl & $\left.\mathrm{C}_{2} \mathrm{C}_{6} \mathrm{H}_{3} \mathrm{CH}_{2}\right)_{3}$ & 0.7788 & 4.2 & 5.32 \\
benzyl & $\mathrm{C}_{5} \mathrm{H}_{5}$ & 0.7684 & 4.6 & 9.03 \\
phenalene & $\mathrm{C}_{7} \mathrm{H}_{7}$ & 0.7812 & 5.7 & 6.65 \\
allyl & $\mathrm{C}_{13} \mathrm{H}_{9}$ & 0.7977 & 6.6 & 4.99 \\
pentadienyl & $\mathrm{C}_{3} \mathrm{H}_{5}$ & 0.7789 & 7.2 & 9.03 \\
heptatrienyl & $\mathrm{C}_{5} \mathrm{H}_{7}$ & 0.7935 & 7.9 & 6.54 \\
nonatetraenyl & $\mathrm{C}_{7} \mathrm{H}_{9}$ & 0.8071 & 8.1 & 5.15 \\
undecapentaenyl & $\mathrm{C}_{9} \mathrm{H}_{11}$ & 0.8210 & 8.3 & 4.23 \\
\hline
\end{tabular}


Table 2. Summary of energy increments (in au) for twisted ethylene, twisted styrene, and spiro-bis-pentadienyl diradical at their BS $m_{s}=0$ geometry.

\begin{tabular}{llrrr}
\hline energy contributions & notations & $\begin{array}{c}\text { twisted } \\
\text { ethylene }\end{array}$ & $\begin{array}{r}\text { twisted } \\
\text { styrene }\end{array}$ & $\begin{array}{l}\text { spiro-bis- } \\
\text { pentadienyl }\end{array}$ \\
\hline triplet restricted & & -78.50913 & -309.63596 & -425.16935 \\
frozen-core $m_{s}=0$ & ${ }^{3} E_{R}$ & -78.50847 & -309.63552 & -425.16845 \\
frozen-core singlet & $E_{a \bar{b}}$ & -78.50782 & -309.63508 & -425.16755 \\
direct exchange integral & ${ }^{1} E_{R}$ & 0.00066 & 0.00044 & 0.00090 \\
triplet $m_{s}=1$ unrestricted & $K_{a b}$ & -78.51112 & -309.63960 & -425.17644 \\
BS $m_{s}=0$ unrestricted & ${ }^{3} E_{U}$ & -78.51250 & -309.64056 & -425.17519 \\
spin polarization from monoexcitations for $m_{s}=1$ & $E_{U, a \bar{b}}$ & -0.00200 & -0.00364 & -0.00710 \\
spin polarization from monoexcitations for $m_{s}=0$ & ${ }^{3} E_{U}-{ }^{3} E_{R}$ & -0.00402 & -0.00504 & -0.00674 \\
total spin polarization for triplet & $E_{U, a \bar{b}}-E_{a \bar{b}}$ & -0.00801 & -0.01232 & -0.02094 \\
total spin polarization for singlet & ${ }^{3} E_{S P}^{(2)}$ & -0.01207 & -0.01512 & -0.02023 \\
spin-decontaminated triplet & ${ }^{1} E_{S P}^{(2)}$ & -78.51714 & -309.64828 & -425.19028 \\
spin-decontaminated singlet & $E_{T}$ & -78.51989 & -309.65020 & -425.18868 \\
S-T gap before spin decontamination ${ }^{\mathrm{a}, \mathrm{b}}$ & $E_{S}$ & 0.9 & 0.6 & -0.8 \\
S-T gap after spin decontamination ${ }^{\mathrm{a}, \mathrm{b}}$ & $\Delta E_{S T, U}$ & 1.7 & 1.2 \\
energy of closed-shell planar minimum & $\Delta E_{S T}$ & -78.61398 & -309.72721 \\
torsional barrier before spin decontamination ${ }^{\mathrm{a}}$ & & 63.7 & 54.4 & -1.0 \\
torsional barrier after spin decontamination ${ }^{\mathrm{a}}$ & & 59.0 & 48.3 & \\
\hline
\end{tabular}

a In kcal/mol.

${ }^{\mathrm{b}}$ Positive sign indicates a singlet ground state. 


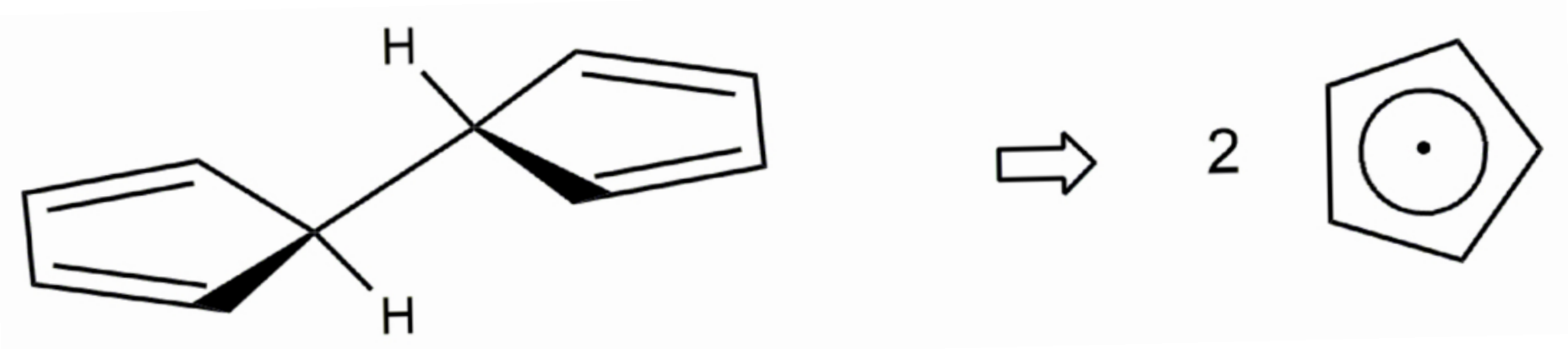



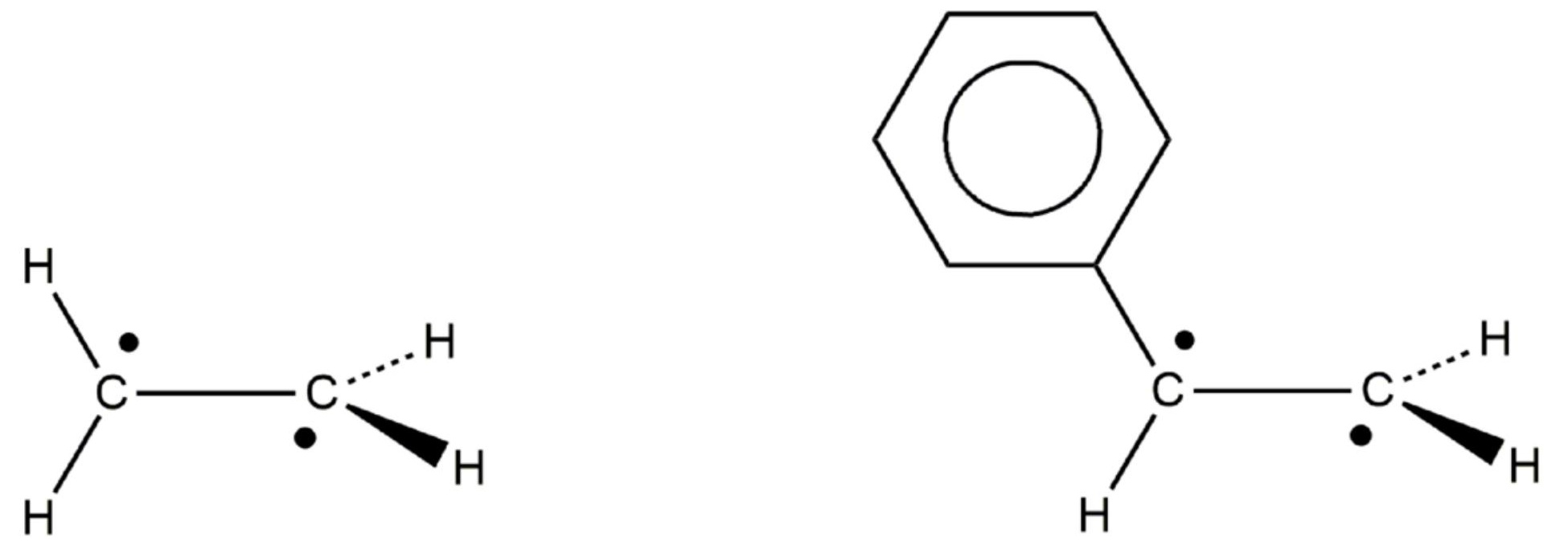


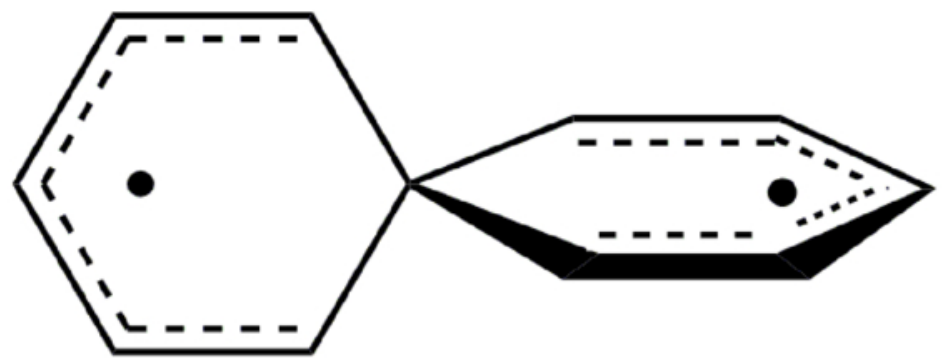



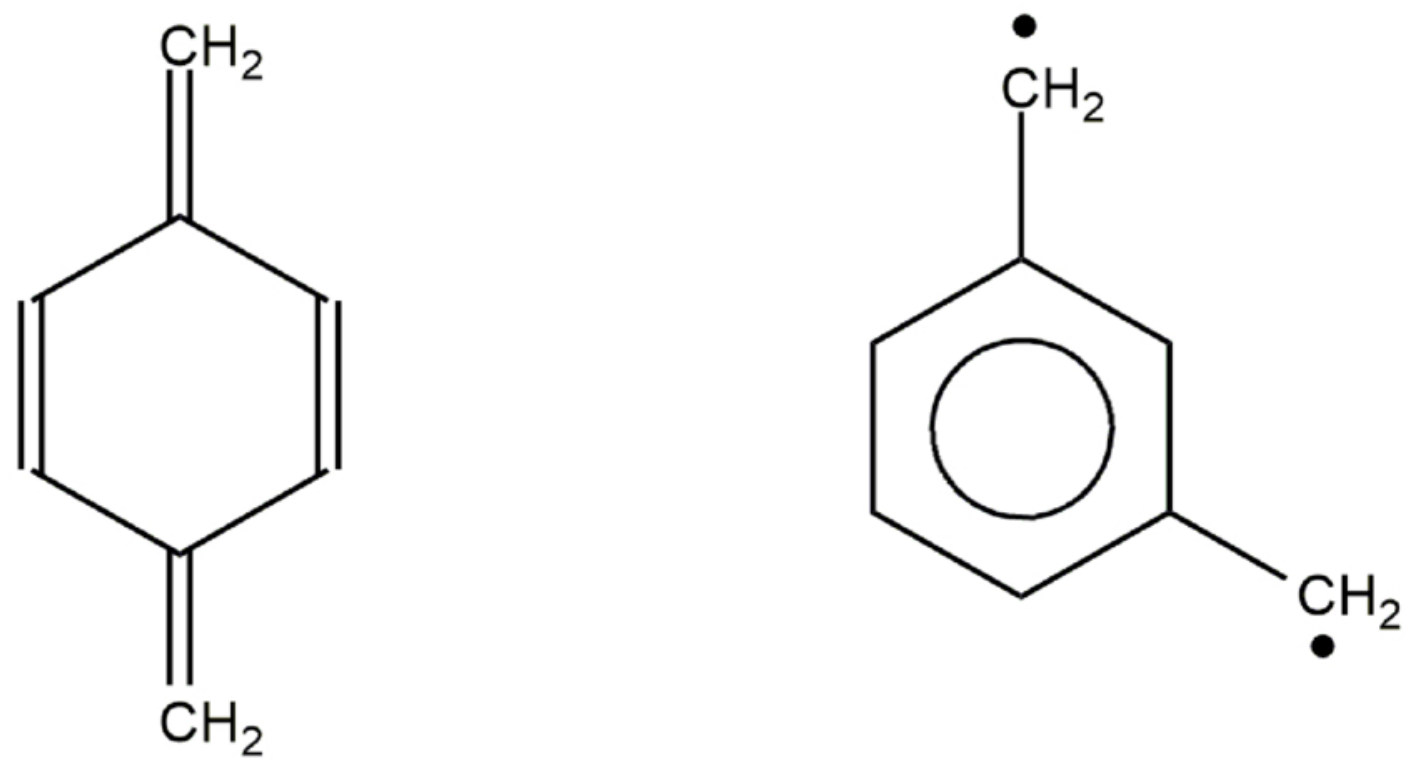

para 